\title{
LCG MCDB - Knowledge Base of Monte Carlo Simulated Events
}

\author{
Sergey Belov ${ }^{1}$ \\ Joint Institute for Nuclear Research \\ Dubna, Moscow region, Russia, 141980 \\ E-mail: Sergey.Belov@jinr.ru

\section{Lev Dudko} \\ Scobeltsyn Institute of Nuclear Physics of Lomonosov Moscow State University \\ Moscow, Russia, 119992 \\ E-mail:dudko@fnal.gov
}

\section{Anton Gusev}

Institute For High Energy Physics

Protvino, Russia, 142281

E-mail: Anton.Gusev@cern.ch

\section{Witold Pokorski}

\section{CERN/SFT}

CH-1211 Geneva 23, Switzerland

E-mail: Witold.Pokorski@cern.ch

\section{Alexander Sherstnev \\ Cavendish Laboratory, University of Cambridge \\ CB3 OHE, UK \\ E-mail: cherstn@hep.phy.cam.ac.uk}

In this paper we report on LCG Monte Carlo Data Base (MCDB) and software which has been developed to operate MCDB. The main purpose of the LCG MCDB project is to provide storage and documentation system for sophisticated event samples simulated for the LHC collaborations by experts. In many cases, the modern Monte Carlo simulation of physical processes requires expert knowledge in Monte Carlo generators or significant amount of CPU time to produce the events. MCDB is a knowledgebase mainly dedicated to accumulate simulated events of this type. The main motivation behind LCG MCDB is to make the sophisticated MC event samples available for various physical groups. All the data from MCDB is accessible in several convenient ways.

LCG MCDB is being developed within the CERN LCG Application Area Simulation project.

XI International Workshop on Advanced Computing and Analysis Techniques in Physics Research Amsterdam, the Netherlands

23-27 April, 2007

$1 \quad$ Speaker 


\section{Introduction}

The LCG MCDB project [1,2] has been created to facilitate communication between experts/authors of Monte Carlo (MC) generators and users of the programs in the LHC collaborations.

The current version of LCG MCDB provides flexible infrastructure to share samples of events of particle collisions in accelerators prepared by the MC method (MC event samples) and keep the files in a reliable and convenient way. It has several interfaces, mainly Web-based, which help to carry out routine operations with stored samples by users and authors of the samples.

LCG MCDB is particularly useful in tasks where the preparation of event samples requires specific knowledge of the Monte-Carlo codes/techniques applied, significant computing power, and/or constant interaction between end-users and the authors of the events. In many standard tasks events can be produced "on the fly" keeping just initial "data-cards", i.e. MC code parameter values which unambiguously define a concrete simulation run. But if the simulation time, or exploitable resources, becomes a significant factor, it would be worth considering the event sample as a candidate to keep in LCG MCDB. For instance, this situation can arise if we use such MC programs as ALPGEN [3], CompHEP [4], GRACE [5], or MadGraph [6]. Even MC generators as PYTHIA or HERWIG sometimes require the keeping of event files themselves. Examples of this sort happen in simulations of rare processes and/or with strong pre-selection cuts.

The second motivation behind the project is to create a central database of MC events where stored event samples are publicly available for all groups to use and/or validate. Often, different groups of experimentalists prepare similar event samples or turn to the same group of authors of MC generators in the simulation. For example, the same MC samples of Standard Model (SM) processes can be employed for the investigations either in the SM analyses (as a signal) or in searches for new phenomena in Beyond Standard Model analyses (as a background). Publicity of the event samples equipped with corresponding and comprehensive documentation can speed up cross checks of the samples themselves and physical models applied. It also prevents possible waste of researcher time and computing resources.

The previous version [7] of MCDB was launched by the CMS collaboration in 2002. Several years of extensive use of the database have shown some limitations of a design applied in CMS MCDB. Storage of event files on $\mathrm{AFS}^{2}$ allows one to keep only small sized MC samples (basically parton level events prepared by Matrix Element tools), phonetic-based search turned out to be insufficient, and the database does not have simple tools to reuse information entered in MCDB earlier, such as physical parameters (masses, couplings, etc.), process information (name, PDF, particle content), generator information, etc. The new design of MCDB presented in this paper overcomes these problems and gives opportunities for further development of the idea. LCG MCDB is based on much more powerful, standardized and

\footnotetext{
The Andrew File System (AFS) is a distributed networked file system developed by Carnegie Mellon University as part of the Andrew Project.
} 
exportable software tools that are available to the LHC collaborations. Current migration of CMS physical groups from old CMS MCDB to the LCG framework gives us the motivation for the further development of the tool.

In the next sections we describe the LCG MCDB design and ideas in more detail and briefly portray subsystems and modules of LCG MCDB. Section 4 reports how end-users can use the software. A more detailed manual and installation instructions are available on the LCG MCDB server ([8], help section).

At present, LCG MCDB is a stable software package and ready to use for the LHC community. A dedicated web server is deployed in [8].

\section{LCG MCDB as a knowledgebase}

Knowledgebase is a special kind of database for knowledge management. It provides the means for the computerized collection, organization, and retrieval of knowledge [9]. According to the definition one of the specific features of knowledgebase is that it keeps metadata or metainformation, i.e. information on data. Usually it is not possible to strictly distinguish between data and metadata, since the separation depends on situations where the data are exploited. In ur concrete case we discriminate between events, as sets of particle 4-momenta (data), and information describing the events as one entity, an event sample (metadata). The latter is also not very strictly defined. For example, if the number of particles in an event is the same for the whole sample, we can add the parameter to metadata. But if it varies from event to event it is certainly a part of data. In our definition of metadata we try to single out the most common characteristic of event samples, which could be applied in most cases. Metadata form the main contents of MCDB. In this sense, MCDB can hold a path to an event sample only and the sample itself can be located somewhere else.

The benefit of the separation is the following. MCDB interfaces provide the means to manipulate with metadata only ${ }^{3}$. This simplifies the structure and software of MCDB drastically. Thus by means of MCDB interfaces a user can search for a necessary event sample (according to given criteria) and let the user know what the sample holds and how the events were prepared. In other words the user should know how to reproduce the events. According to the idea the metadata must describe the corresponding event sample in a comprehensive manner. This information should be entered by the event sample author. In some cases, metadata are encoded inside the event file itself and can be inserted to MCDB (semi-) automatically. We postpone a discussion of this case until the Section 6.

Comprehensive description of an event sample requires a lot of information, which should be entered to the database. However, in practice, in this specific application area a large part of the information is common for lots of samples. For example, the Standard Model processes are described by a large set of SM couplings and particle parameters (masses, widths, etc.) ${ }^{4}$, but usually, only few parameters are modified from one sample to another. In the MCDB

3 Except for interfaces which are responsible for downloading and uploading of event files. See more details in the next sections.

4 A worse situation arises in Beyond SM models, where we can have hundreds of physical parameters in some models. 
conception we introduce "Model" - a set of parameters, which can be attached to an event sample. The user can choose an appropriate model and change a few parameters in the model and store the modified set of parameters as a new model with a new name. The same solution is used in the description of MC generators. We introduce a standard record to describe the programs. The user simply chooses one of the standard records and attaches it to a new article. In order to include the features in the author interface we developed our own Content Management System (CMS) with a flexible structure, which can be extended in a simple manner.

The second idea behind the current design of MCDB is that MCDB is an area for interaction between two different communities, producers of events and consumers of the events. The latter users are end-users and the former users are "authors". Since the goals and tasks of the two groups are different, the interfaces intended for each group of users are also different. Any physicist who feels his/her sample is worthy to come within MCDB can make a request to open an author account on the MCDB server. It means MCDB does not assume to have a special team (of event producers) to prepare events according to end-user requests.

There are several blocks in LCG MCDB, which should be realized:

- Content Management System (CMS) with a powerful and flexible Web interface for authors of event samples. It should have several types of templates to simplify the task of event sample description.

- A block of tree graph of physical categories with articles published by authors. This is the main part MCDB visible via Web browsers with no authorization in MCDB.

- A powerful search engine based on SQL/XML to search for contents of MCDB.

- A programming interface to CASTOR [10], which is used as a native storage of event samples.

- A block of direct uploading of files from Web/AFS/CASTOR/Grid to MCDB.

- Block of direct downloading of files from MCDB via Web/CASTOR/Grid (URI).

- A flexible and reliable authorization system based on CERN AFS/Kerberos logins or LCG Grid certificates

- Backup system for all stored samples and corresponding SQL information.

- API to the LHC collaboration software environments.

- The standard record of an event sample. The record should be encoded to a set of SQL tables.

- A unified and flexible format of event files based on the LHEF agreement and the HepML language. A programming package which supports the format.

In general, metadata can hold very specific information and can be presented in an arbitrary form. In fact, it is one of the main problems of knowledge bases, since the arbitrariness results in problems in introducing effective and relevant search methods in knowledge bases. Our situation is simpler than the general case and we can limit ourselves by some general set of parameters which cover most parts of the necessary information on event samples. Owing to specific purposes and application area of MCDB, we can define the standard record for MCDB articles. Now the record corresponds to a set of parameters stored as a record in our relational DB and a comment written by the author in free form. All information which is not kept within 
the standard record can be and should be put in the comment. MCDB search requests use the standard records to retrieve information. If MCDB users, authors or end-users, request to add new parameters to the standard record it can be extended.

The standard information to describe event samples can be divided into several blocks. Each of the blocks corresponds to a definite set of parameters which are necessary to interpret a concrete event sample. The list below gives a short description of the main blocks:

- General information about a simulated event sample or a group of samples

o Title of physical process

o Physical Category (e.g. Higgs, Top physics or $\mathrm{W}+$ jets processes)

o Abstract (short description)

o List of authors

o Name of an experiment and/or a group (for which the sample was prepared or intended)

o Author comment on the sample (some additional unstructured information on the sample)

- Physical process

o Initial state (names of beam particle, energy, etc.)

o Final state (name of the final particles, etc.)

o QCD scale(s)

o Process PDF (parton distribution functions) applied

o Information on separate subprocesses, if they are distinguished

o Event file

o File name

o The number of events

o Cross section and cross section error(s)

o Author comment

- Used MC generator

o Name and version

o Short description

o Home page Web-address

- Theoretical model used to simulate the events

o Name

o Short Description

o A set of physical parameters and their values with the author's descriptions

o Applied cuts

\section{LCG MCDB Software Description}

This section describes shortly all subsystems and software technologies adopted in LCG MCDB. The current version of LCG MCDB is based on the following technologies: WWW, CGI, Perl, SQL, XML, CASTOR, and Grid. MCDB is a Web server written as a set of Perl CGI scripts. The scripts interact with relational DB by means of SQL requests and can generate either Web pages or XML documents. The main storage of event files is based on tape robots at 
CERN available via CASTOR. The MCDB software is organized as a set of Perl modules with the possibility of installing and customizing the software on other sites. All of the MCDB software has been developed from scratch and is available publicly in LCG CVS [11].

For the whole contents of LCG MCDB we provide a daily backup of the SQL DB and double mirroring of the samples in CASTOR. The main unit of MCDB is an article, a document describing one or several event samples. MCDB articles are distributed into categories, i.e. a set of articles concerning a particular type of physical process (e.g. top physics, Higgs physics) or theoretical model (e.g. supersymmetry, extra dimensions). Each category has its own branch in the main MCDB Web tree graph. The access system in MCDB reminds of a classical system used in the usual Internet forums or newsgroups. There are four different types of permissions to access MCDB. The end-user access is reserved for users who are interested in requesting a new event sample or in downloading or making comments to already published event samples. The author access is reserved for authorized users (MC experts) and requires registration on the main Web site. Only authors can upload and describe new event samples. The moderator access is reserved for users who manage author profiles and are responsible for general monitoring of information uploaded. The administrator access is reserved for software developers and maintainers who take care of the database itself. The scheme of LCG MCDB is shown in Fig. 1.

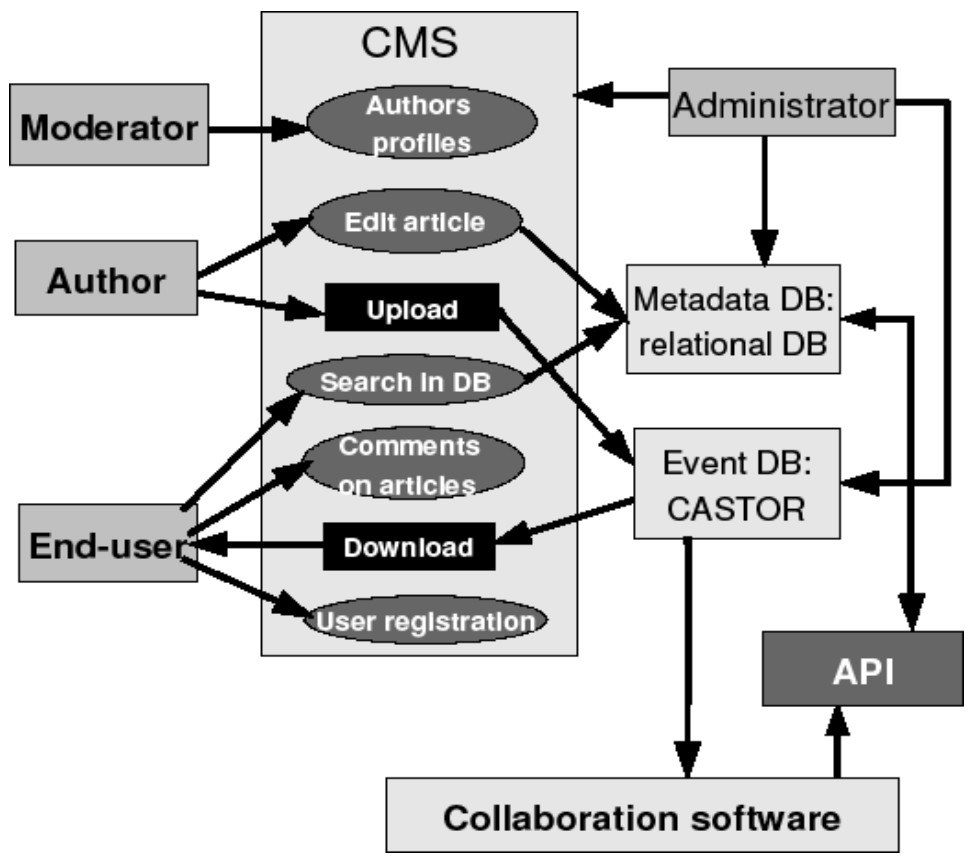

Fig. 1 This general scheme of LCG MCDB shows main interfaces and interaction with main consumers

\subsection{Web interface}

The Web-interface consists of two parts:

- End-user area, where any user can search for a necessary event sample in the whole set of available samples. Requests can be done either via a search form or 
by browsing the main tree graph of categories where all articles are available. Users can read the description of the events, download the samples, ask questions about the samples and read the previous discussions on the particular event sample.

- Author area, where authorized authors can upload new event files to the database and describe the events using the MCDB template system. As we mentioned, the user does not need to enter all the necessary information from scratch, since the templates have a lot of pre-entered information. Authors can interact with the end-users of their samples on public forums attached to each article. With the same interface, authors can edit his/her own previous articles or make the articles temporarily inaccessible in the end-user area.

\subsection{SQL DB}

LCG MCDB adopts MySQL. The SQL technology provides a possibility to keep information in a very structured way. Authors provide documentation on event samples through forms and MCDB scripts translate the information to records in the MCDB relational database.

\subsection{Search engine}

Since we use a relational DB, it is possible to provide a variety of complex search queries, which can use specific relations between DB records. The deployed Web search interface is realized as a dynamic query construction wizard which is based on the JavaScript XML-query constructor. The development of application programming interfaces to specific external software (for example a simulation framework of a LHC experiment) may benefit from similar tools in order to simplify the query construction.

\subsection{Storage}

As a native storage interface for event samples we have selected CASTOR, because of the absence of serious space limitations on tapes and taking into account popularity of the interface in the LHC collaborations. We provide direct CASTOR paths for all LCG MCDB samples and also several options to obtain the samples through other interfaces (HTTP, GridFTP etc.). A local disk cache system is used to speed up the storage operations.

\subsection{Authentication and authorization}

We pay special attention to the security of transactions during all LCG MCDB operations. All of the transactions are encrypted by SSL technology. There are two ways to log in to MCDB. The first one relies on CERN AFS/Kerberos login/password. The second mechanism uses LCG Grid certificates. Authors can choose either or both of these ways. These authorization methods are standard at CERN and any CERN user can use at least one of these two methods.

\subsection{Documentation}

Most of the LCG MCDB documentation is available from the dedicated web server. The information consists of different parts. Technical documentation describes the current 
implementation of LCG MCDB itself. The user documentation is organized as a set of How-To for users and authors. A separate documentation (available from the CVS repository) is intended for developers of the LCG MCDB software. A brief start-up manual for non-experienced LCG MCDB users is also available in the next section of this document. In addition, there are two freely accessible mailing lists dedicated to users and developers. Their addresses are available in the documentation section on the main web page of the server.

\section{How to use LCG MCDB}

A user who is going to find and download events for a particular process can browse the MCDB categories and subcategories (the menu at the left side of the main LCG MCDB web page [8]) and verify, whether an appropriate sample has already been generated. If this is the case, the user may read the sample article describing how the events are prepared (check parameters of the theoretical model, generator name and generation parameters, kinematic cuts, etc.). At the bottom of the page there is a link to the uploaded file(s), as well as a direct CASTOR path to the sample. The web page also contains a link to the "Users Comments" interface, where users can ask questions about the sample and browse the previous discussions on the article. Users do not need any authorization to archive all the steps described above. The search engine provides different possibilities of search queries based on the set of main parameters of the article and samples.

If a user wants to upload a new event sample or publish a new article in LCG MCDB (it means the user will become an author), (s)he should follow the following procedure:

- Register as a new author. There is a link to the registration interface on the right side menu of the main MCDB Web page. Wait for a confirmation e-mail.

- Login to the LCG MCDB authors area.

- Choose the option "Create New Article" in the authors menu. It appears at the right side after authorization.

- Fill all necessary forms in the documentation template (title, generator, theoretical model, cuts, etc.)

- Upload event files in the "Event Files" sub-window.

- Tick the box "Publish" and click "Preview/Save".

As we mentioned above for authorization the user needs a valid CERN AFS login or a LCG GRID digital certificate.

Authors can save unfinished articles in MCDB and resume to edit them later. Authors can edit their previous articles that are already published on the Web or make the articles publicly inaccessible for a while.

The LCG MCDB team appreciates any bug reports, feedback, comments or suggestions for possible new implementations and improvements of the service (LCG MCDB).

\section{API to collaboration software}

Apart from the MCDB server, LCG MCDB team provides Application Programming Interfaces (APIs) specific to the simulation environments of the LHC collaborations. The main 
idea of these subsystems is to develop a set of routines for the collaboration software which would give a direct access to the LCG MCDB files during the MC production on computer farms.

The simplest way to access event samples is to use direct WEB, CASTOR or Grid path to the event samples. This way does not require any special software developments on the side of collaboration software. This way, however, does not provide any possibility for automatic access to event sample description. This is the reason we developed a more complicated interface which could be used for automatic processing of event samples and the corresponding documentation. According to our idea, MCDB team provides API based on XML representation of event sample metadata. The current version of the API is a C++ library, which can be added to collaboration software. The XML output from LCG MCDB is based on the HepML [12],[13] specifications (for more details, see the next section).

The current library contains $\mathrm{C}++$ classes and provides routines to fill the class objects with information from a MCDB article, including CASTOR/Grid/HTTP paths to event files attached to the article. Such an interface has already been implemented in the CMS collaboration software environment. The software and documentation are available in LCG CVS [11].

On the Fig. 2 is represented a scheme of interaction between user software and MCDB with the aid of API.

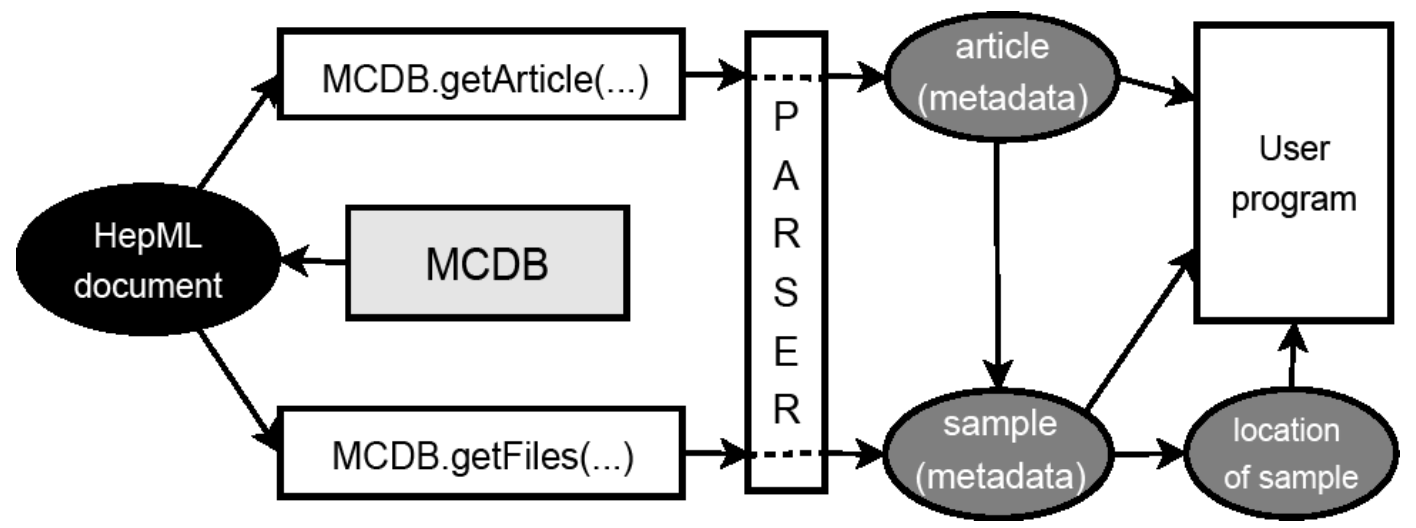

Fig. 2This general scheme shows how the API interacts with LCG MCDB to external user software

In the future, some emphasis will be put on the development of extensions of API specific to the automatic uploading of HepML information and event samples to MCDB. This development will be carried out in the context of the HepML project.

\section{A unified XML format HepML}

At present, each MC generator supports its own output format of event files. Authors of Matrix Element tools provide interface programs to pass the events of a particular MC generator to the subsequent level of simulation (i.e. showering, hadronization, decays, simulation of detector response). The first step to standardize such interfaces has been described in the agreement "Les Houches Accord Number One” (LHA-I) [15], where a definite and strict structure of FORTRAN COMMON BLOCKS to transfer the necessary information from one 
code to another was fixed. The second step in this direction has been done in the agreement "Les Houches Event File" (LHEF) [16], where the information fixed in LHA-I is used in the event file structure. All other information can be kept in a specific place inside the header of the event file. The standard does not apply any limitations on the extra information and the structure of the block. The next natural step is to provide a unified format to keep the necessary information within the LHEF structure. In this context the other information means the metadata described in the Section 2 and some other information specific to the sample (parameters of matching in different schemas, information on specific NLO approximations, jet parameters, etc.).

Owing to the highly dissimilar nature of the information, the most appropriate technology for the unified representation could be XML-based format. In this case it can provide the possibility to describe the stored information in a very flexible and structured way.

The main idea behind the XML-based format is the flexibility to build and include a set of necessary parameters in an event file. For example, different MC generators may use the same tags for description of the physical parameters or they may need to keep specific information (through introduction of new dedicated tags). The new tags do not spoil the event file format and we do not need to re-write our routines which process these event files automatically. HepML is now being developed within the special LCG HepML project in collaboration with the CEDAR project [13]. More information is available on our Wiki page [14].

As the first part of HepML we have prepared several XML Schemas. The main goal of the schemas is to provide a general and formal description of event data structures which are kept in XML files. Authors of MC codes can use the XML Schemas in developing of I/O routines. If the routines are consistent with the schemas, event files generated be the routine can be read by other programs without changes in input routines of the programs. Also the schemas can be used for validation of event files if the files are written according to HepML specifications. Now we have three main schemas. The first XML Schema lha1.xsd corresponds to the whole set of parameters composing the LHA-I agreement. The other two schemas, sample-description.xsd and mcdb-article.xsd, describe parameters which are necessary to generate an XML data for an event sample and to form an LCG MCDB article for the sample. It means it includes all parameters mentioned in Section 2 except for some parameters from LHAI. The CEDAR team develops other XML Schemas for other tasks arising in the problem of automatization of data processing in HEP. Now all the schemas are unified in one general formal XML Schema hepml.xsd, which includes all the other schemas as sub-schemas. This solution leaves freedom to develop schemas and software in independent groups, but to use schemas of both groups in one software project. All the schemas are available in [17].

Possible internal adaption of LHEF and HepML formats into the most popular MC generator projects would result in a significant improvement of the MC event sample documentation and book-keeping. Such adaption of unified standards provides the possibility to develop new standard interfaces and utilities.

The LCG MCDB project already implements a part of HepML specifications in MCDB API. A dedicated document discussing the details of the requirements and describing the HepML proposal will appear in the near future [18]. 


\section{Conclusion}

MCDB is a special knowledgebase designed to keep event samples for the LHC experimental and phenomenological community. Now, a new version of the software has been finished and the server is ready for use by the community. Some new important features are implemented in the software. The features simplify and improve the process of documentation of event samples.

In addition to the server, MCDB team has prepared an API for the LHC collaboration software environments. Implementation of the API to the software environments could give a possibility to use MCDB as a native storage in large-scale productions in collaborations.

Subsequent development of the software will rely on further standardization of event file formats and elaboration of the HepML specifications and software.

\section{Acknowledgements}

This work was partially supported by the RFBR (the RFBR grant 07-07-00365-a). We thank Seyi Latunda-Dada for discussions of the text. We also acknowledge the LCG collaboration for support and hospitality at CERN. Participation of A. S. in the project was partly supported by the UK Particle Physics and Astronomy Council.

\section{References}

[1] M. Dobbs et al., The QCD/SM working group: Summary report, Proceedings of Les Houches Physics at TEV Colliders 2003 [arXiv:hep-ph/0403100].

[2] P. Bartalini, L. Dudko, A. Kryukov, I. V. Selyuzhenkov, A. Sherstnev and A. Vologdin, LCG Monte-Carlo data base [arXiv:hep-ph/0404241].

[3] M. L. Mangano, M. Moretti, F. Piccinini, R. Pittau and A. D. Polosa, ALPGEN, a generator for hard multiparton processes in hadronic collisions, JHEP 0307, 001 (2003) [arXiv:hep-ph/0206293].

[4] E. Boos et al. [CompHEP Collaboration], CompHEP 4.4: Automatic computations from Lagrangians to events, Nucl. Instrum. Meth. A 534, 250 (2004) [arXiv:hep-ph/0403113].

[5] F. Yuasa et al., Automatic computation of cross sections in HEP: Status of GRACE system, Prog. Theor. Phys. Suppl. 138, 18 (2000) [arXiv:hep-ph/0007053].

[6] F. Maltoni and T. Stelzer, MadEvent: Automatic event generation with MadGraph, JHEP 0302, 027 (2003) [arXiv:hep-ph/0208156].

[7] L. Dudko, A. Sherstnev, CMS MCDB http://cmsdoc.cern.ch/cms/generators/mcdb

[8] S. Belov, L. Dudko, A. Gusev, A. Sherstnev, LCG MCDB http://mcdb.cern.ch

[9] http://en.wikipedia.org/wiki/Knowledge_Base

[10] J. P. Baud, B. Couturier, C. Curran, J. D. Durand, E. Knezo, S. Occhetti and O. Barring, CASTOR status and evolution, In the Proceedings of 2003 Conference for Computing in High-Energy and 
Nuclear Physics (CHEP 03), La Jolla, California, 24-28 Mar 2003, pp TUDT007 [arXiv:cs.oh/0305047].

[11] MCDB project page on LCG Savannah portal http://savannah.cern.ch/projects/mcdb

[12] A. Sherstnev, HEPML: proposal for a structure of partonic events files http://agenda.cern.ch/fullAgenda.php?ida=a035826 ; TWiki Page https://twiki.cern.ch/twiki/bin/view/Main/HepML

[13] CEDAR HepML Page http://projects.hepforge.org/hepml/

[14] CEDAR HepML Wiki http://projects.hepforge.org/hepml/trac/wiki, LCG HepML Wiki http://twiki.cern.ch/twiki/bin/view/Main/HepML

[15] E. Boos et al., Generic user process interface for event generators, [arXiv:hep-ph/0109068].

[16] J. Alwall et al., A standard format for Les Houches event files, Comput. Phys. Commun. 176, 300 (2007) [arXiv:hep-ph/0609017].

[17] S. Belov, L. Dudko, A. Sherstnev, HepML XML Schema http://mcdb.cern.ch/hepml/schemas/

[18] CEDAR team and LCG MCDB team, HepML: XML-based description language for documents by Monte Carlo generators, in progress. 